\title{
Glypican-1 immunohistochemistry does not separate mesothelioma from pulmonary adenocarcinoma
}

\author{
Kenrry Chiu ${ }^{1,2} \cdot$ Lawrence Lee $^{1,2} \cdot$ Simon Cheung ${ }^{1,2} \cdot$ Andrew M. Churg ${ }^{1,2}$
}

Received: 8 March 2018 / Accepted: 24 March 2018 / Published online: 21 May 2018

(c) United States \& Canadian Academy of Pathology 2018

\begin{abstract}
Immunohistochemistry (IHC) is used to help differentiate pleural mesothelioma from pulmonary adenocarcinoma in pleural biopsies and cytology specimens of pleural effusions due to overlapping morphologic features between these two malignancies. The aim of this study is to evaluate IHC glypican-1, a recently proposed marker for epithelioid mesothelioma, in our cohort of mesotheliomas and pulmonary adenocarcinoma. Tissue microarrays with duplicate cores from 33 cases of mesotheliomas (28 epithelioid type and five sarcomatoid type) and 21 cases of pulmonary adenocarcinoma were stained with glypican- 1 antibody. The proportion of cases by tumor type showing staining with glypican- 1 and the $H$-score for each tumor type were evaluated. All 33 cases of mesothelioma and all 20 cases of pulmonary adenocarcinoma with interpretable cores showed positive cytoplasmic staining. All but one case of mesothelioma and all pulmonary adenocarcinomas showed staining in at least $80 \%$ of the tumor cells. The mean $H$-score for glypican- 1 of mesothelioma $(134 \pm 59$, mean \pm SD) was not significantly different from that for pulmonary adenocarcinoma $(156 \pm 60 ; P=0.21)$. Neither epithelioid type (mean $H$-score $135 \pm 57$ ) nor sarcomatoid type (mean $H$-score $130 \pm 78$ ) of mesothelioma showed different $H$-scores when compared to pulmonary adenocarcinoma $(P=0.23$ and 0.42 , respectively). In conclusion, glypican-1 IHC does not differentiate mesothelioma from pulmonary adenocarcinoma.
\end{abstract}

\section{Introduction}

A commonly encountered problem with pleural biopsies or cytology specimens of pleural effusions is the distinction between pleural mesothelioma and pulmonary adenocarcinoma [1-3]. Both entities feature a proliferation of atypical epithelioid cells. Mesotheliomas can show growth patterns that mimic adenocarcinoma, including tubulopapillary, acinar, and adenomatoid patterns. Because of the overlapping morphologic features between mesothelioma and carcinoma, immunohistochemistry (IHC) is frequently used to establish the diagnosis. However, most individual immunochemical markers are not entirely specific, so it is generally recommended that a panel of immunohistochemical stains that

Andrew M. Churg

achurg@mail.ubc.ca

1 Department of Pathology, Vancouver General Hospital, Vancouver, BC, Canada

2 Department of Pathology, University of British Columbia, Vancouver, BC, Canada includes at least two mesothelioma markers and two carcinoma markers be used in this setting [1-7]. Thus, an immunochemical marker that is only expressed on mesothelial cells would be diagnostically very valuable.

Glypicans are cell-surface-associated proteoglycans that bind signaling factors affecting cell proliferation, mobility, and adhesion, and are upregulated in a variety of tumors [8 and see Discussion]. Recently, Amatya et al. reported that IHC with glypican-1 is a highly sensitive marker for epithelioid mesothelioma, and only rarely stains pulmonary adenocarcinoma [9]. Glypican-1 was therefore proposed as a highly sensitive and specific marker for epithelioid mesothelioma when considering pulmonary adenocarcinoma in the differential diagnosis. The aim of this study is to evaluate glypican-1 as an immunohistochemical marker in our cohort of mesotheliomas and pulmonary adenocarcinoma.

\section{Materials and methods}

Tissue microarrays of duplicate $0.6 \mathrm{~mm}$ cores were constructed from formalin-fixed, paraffin-embedded tissue 
Fig. 1 Representative images of a an adenocarcinoma and b mesothelioma with both showing diffuse staining of moderate intensity for glypican-1
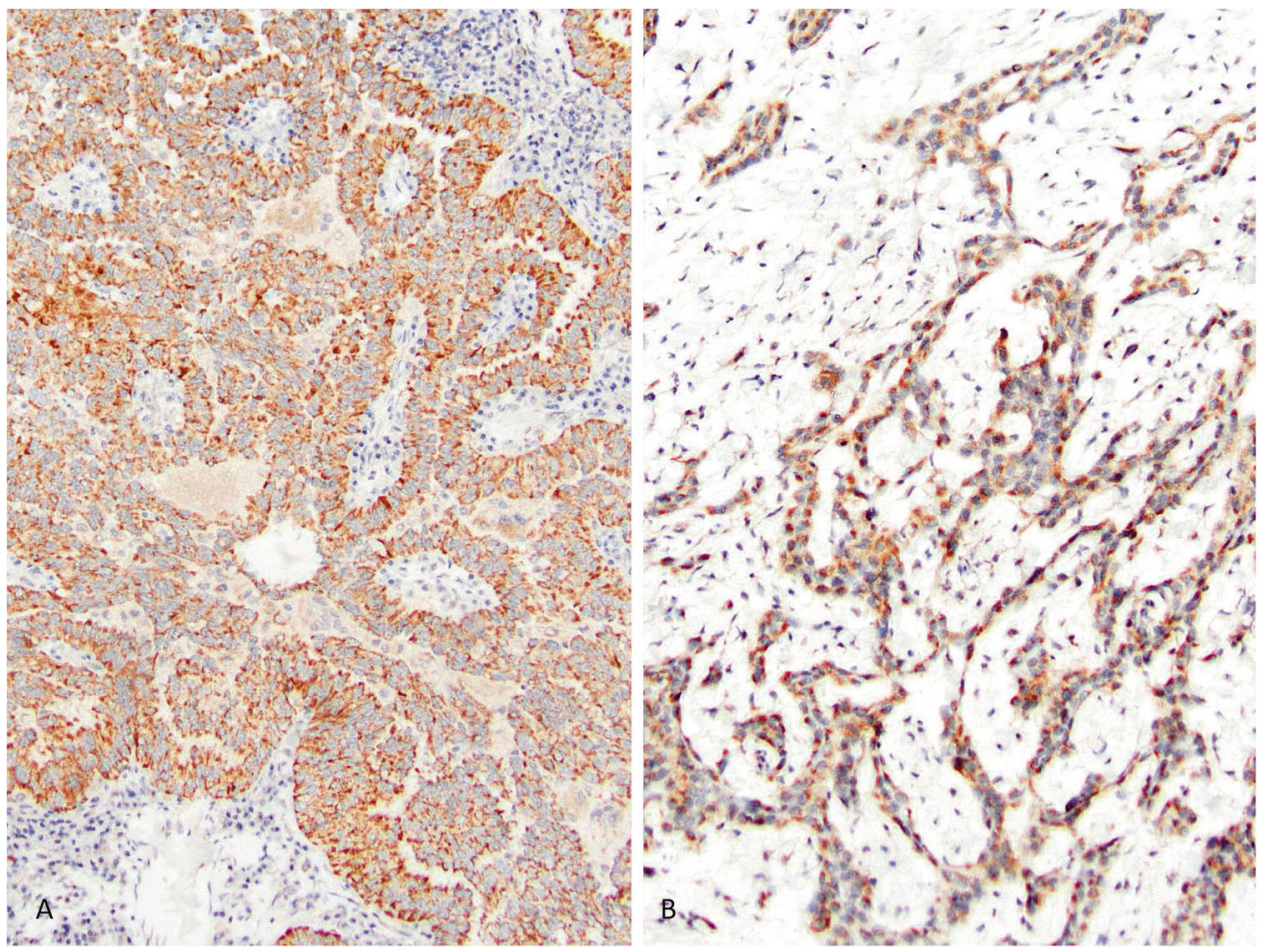

blocks from 31 cases of mesotheliomas (28 epithelioid and five sarcomatoid type), 19 reactive mesothelial proliferations, and 21 cases of pulmonary adenocarcinomas from the surgical pathology files at Vancouver General Hospital. Duplicate cores of 19 cases of benign reactive mesothelial proliferation were also included. Approval from the institutional ethics board was obtained for this study.

IHC was performed on the tissue microarrays with glypican-1 rabbit polyclonal antibody (1:100 dilution; Proteintech Group, Rosemount, IL, USA), the same antibody and dilution used by Amatya et al. [9]. Heat-induced epitope retrieval was performed for $30 \mathrm{~min}$ with EnVision FLEX TRS high pH buffer (Agilent Technologies, Santa Clara, CA, USA). The staining protocol included 20 min of incubation with the primary antibody, $10 \mathrm{~min}$ of incubation with EnVision FLEX+ Rabbit Linker, and 20 min of incubation with the labeled polymer EnVision FLEX/HRP.

Glypican-1 IHC was scored semi-quantitatively by one pathologist (KC) using an $H$-score, which is obtained by multiplying the proportion of cells showing cytoplasmic staining and the intensity of cytoplasmic staining ( 0 , no staining; 1 , weak; 2 , moderate; and 3 , strong). The core with the higher $H$-score was used for analysis. A case was considered to show positive staining when at least $1 \%$ of tumor cells showed cytoplasmic staining.

The proportion of positive cases for tumor or tissue type, and $\mathrm{H}$-scores for mesothelioma, pulmonary adenocarcinoma, and benign reactive mesothelial proliferation were assessed. The $H$-scores between tumor or tissue types were compared using $t$-tests. A $P$-value of 0.05 or less was considered significant.

\section{Results}

Glypican-1 IHC was performed and assessed on tissue microarrays of 33 cases of mesothelioma, 21 cases of pulmonary adenocarcinoma, and 19 cases of benign reactive mesothelial proliferation. One case of pulmonary adenocarcinoma was excluded from analysis due to insufficient tumor present in the cores for assessment. Otherwise, all cases of mesothelioma, pulmonary adenocarcinoma, and benign reactive mesothelial proliferation showed positive cytoplasmic staining for glypican-1 (Fig. 1). All but one case of mesothelioma showed at least $80 \%$ of tumor cells with positive staining, while all pulmonary adenocarcinomas showed at least $80 \%$ of tumor cells with positive staining. For each case of benign reactive mesothelial proliferation, at least $80 \%$ of mesothelial cells showed staining. The majority of mesotheliomas, $20(61 \%)$ cases, showed weak staining (Table 1). Eleven (33\%) cases of mesothelioma showed moderate intensity, while two cases showed strong staining. The most common intensity pattern seen in pulmonary adenocarcinoma was moderate, seen in 10 $(50 \%)$ cases. Nine $(45 \%)$ cases showed weak staining, while one case showed strong staining. Fourteen (74\%) cases of benign reactive mesothelial proliferation showed 
Table 1 Glypican-1 immunohistochemistry by tumor type

\begin{tabular}{|c|c|c|c|c|c|c|}
\hline \multirow[t]{2}{*}{ Tumor type } & \multicolumn{4}{|c|}{$\begin{array}{l}\text { Intensity of staining: number of } \\
\text { cases }(\%)\end{array}$} & \multirow[t]{2}{*}{$\begin{array}{l}\text { Mean } H \text {-score } \\
\text { (standard deviation) }\end{array}$} & \multirow[t]{2}{*}{$P$-value ${ }^{\mathrm{b}}$} \\
\hline & 0 & 1 & 2 & 3 & & \\
\hline $\begin{array}{l}\text { Pulmonary adenocarcinoma ( } 20 \\
\text { cases }^{\mathrm{a}} \text { ) }\end{array}$ & $0(0)$ & $9(45)$ & $10(50)$ & $1(5)$ & $156(60)$ & - \\
\hline Mesothelioma (33 cases) & $0(0)$ & $20(61)$ & $11(33)$ & $2(6)$ & $135(59)$ & 0.21 \\
\hline Epithelioid type (28 cases) & $0(0)$ & $16(57)$ & $11(39)$ & $1(4)$ & $135(57)$ & 0.23 \\
\hline Sarcomatoid type ( 5 cases) & $0(0)$ & $4(80)$ & $0(0)$ & $1(20)$ & $130(78)$ & 0.42 \\
\hline $\begin{array}{l}\text { Benign mesothelial proliferation } \\
\text { (19 cases) }\end{array}$ & $0(0)$ & $14(74)$ & $5(26)$ & $0(0)$ & $119(43)$ & 0.03 \\
\hline
\end{tabular}

${ }^{a}$ One case of pulmonary adenocarcinoma on the TMA was excluded from analysis due to insufficient tumor for assessment

${ }^{\mathrm{b}}$ In comparison to pulmonary adenocarcinoma weak staining, while the remainder showed moderately intense staining.

The $H$-score for glypican- 1 of mesothelioma ( $134 \pm 59$, mean $\pm \mathrm{SD}$ ) was not significantly different from that for pulmonary adenocarcinoma $(156 \pm 60 ; P=0.21)$. When looking at the histologic types of mesothelioma individually, neither epithelioid $(N=28$, mean $H$-score $135 \pm 57)$ nor sarcomatoid type $(N=5$, mean $H$-score $130 \pm 78)$ showed different $H$-scores compared to pulmonary adenocarcinoma ( $P=0.23$ and 0.42 , respectively). Benign reactive mesothelial proliferation showed a lower mean $H$-score $(119 \pm 3)$ compared to pulmonary adenocarcinoma $(P=0.03)$, but did not differ significantly from mesothelioma $(P=0.31)$. The median $H$-scores for mesothelioma, pulmonary adenocarcinoma, and benign mesothelial proliferation were 100,170 , and 100 , respectively.

\section{Discussion}

In this study, glypican-1 IHC did not differentiate mesothelioma from pulmonary adenocarcinoma. All cases of mesotheliomas and pulmonary adenocarcinoma showed positive cytoplasmic staining for glypican-1, with a diffuse pattern in almost all cases. The $H$-scores for glypican-1 were comparable between pulmonary adenocarcinomas and mesotheliomas, irrespective of histologic type. Our results differ significantly from those of Amatya et al. [9], who reported that glypican-1 was highly sensitive and specific for epithelioid mesotheliomas when differentiating them from pulmonary adenocarcinoma. They reported that all 82 cases of epithelioid mesotheliomas in their study set were positive for glypican-1, while only 3 (3\%) of 97 cases of pulmonary adenocarcinoma were positive. Of note, 80 (98\%) of their cases of mesothelioma showed staining in at least $10 \%$ of the tumor cells, while only three cases of their pulmonary adenocarcinomas showed positive staining in up to only $10 \%$ of tumor cells. This is in contrast to our finding that all pulmonary adenocarcinomas were positive for glypican-1, with at least $80 \%$ of tumor cells showing staining in each case.

The reason for the discordance in the glypican-1 staining, particularly for pulmonary adenocarcinoma, between our study and that of Amatya et al. is unclear. Both studies used the same glypican-1 antibody and antibody dilution. While we used tissue microarrays in comparison to the use of whole tissue sections by Amatya et al. [9], tumor heterogeneity is unlikely to explain the discrepancy. The diffuse staining observed in essentially all of our cases of pulmonary adenocarcinomas means it is unlikely that focal hotspots of staining are being represented by the cores in the tissue microarrays. Interestingly, cytoplasmic staining in benign mesothelial proliferations was also reported by Amatya et al. [9], which was concordant with the findings in our study. In our study, the $H$-score of benign reactive mesothelial proliferation was significantly lower than that for pulmonary adenocarcinoma, but this is not helpful diagnostically.

Glypican-1 expression by IHC has been described in several tumor types, including gliomas [10], cervical squamous cell carcinoma and adenocarcinoma [11], breast cancer [12], and pancreatic cancer [13, 14], and is associated with a worse prognosis in glioblastomas [15], esophageal squamous cell carcinoma [16], and pancreatic ductal adenocarcinoma [14]. These reports raise caution about the utility of glypican-1 as a diagnostic marker given the wide breadth of tumors that can demonstrate staining.

In summary, glypican-1 IHC, in our hands, does not differentiate mesothelioma from pulmonary adenocarcinoma. An immunostain that is highly sensitive and specific for mesothelioma remains desirable.

\section{Compliance with ethical standards}

Conflict of interest The authors declare that they have no conflict of interest. 


\section{References}

1. Husain AN, Colby TV, Ordonez NG, et al. Guidelines for pathologic diagnosis of malignant mesothelioma: a consensus statement from the International Mesothelioma Interest Group. Arch Pathol Lab Med. 2009;133:1317-31.

2. Husain AN, Colby T, Ordonez N, et al. Guidelines for pathologic diagnosis of malignant mesothelioma: 2012 update of the consensus statement from the International Mesothelioma Interest Group. Arch Pathol Lab Med. 2012;137:647-67.

3. Husain AN, Colby TV, Ordonez NG, et al. Guidelines for pathologic diagnosis of malignant mesothelioma: 2017 update of the consensus statement from the International Mesothelioma Interest Group. Arch Pathol Lab Med. 2018;142:89-108.

4. Yaziji H, Battifora H, Barry TS, et al. Evaluation of 12 antibodies for distinguishing epithelioid mesothelioma from adenocarcinoma: identification of a three-antibody immunohistochemical panel with maximal sensitivity and specificity. Mod Pathol. 2006;19:514-23.

5. Bishop JA, Sharma R, Illei PB. Napsin A and thyroid transcription factor-1 expression in carcinomas of the lung, breast, pancreas, colon, kidney, thyroid, and malignant mesothelioma. Hum Pathol. 2010;41:20-5.

6. Lonardi S, Manera C, Marucci R, et al. Usefulness of claudin 4 in the cytological diagnosis of serosal effusions. Diagn Cytopathol. 2011;39:313-7.

7. Ordonez NG. Value of claudin-4 immunostaining in the diagnosis of mesothelioma. Am J Clin Pathol. 2013;139:611-9.

8. Theocharis AD, Skandalis SS, Tzanakakis GN, et al. Proteoglycans in health and disease: novel roles for proteoglycans in malignancy and their pharmacological targeting. FEBS J. 2010;277:3904-23.

9. Amatya VJ, Kushitani K, Kai Y, et al. Glypican-1 immunohistochemistry is a novel marker to differentiate epithelioid mesothelioma from lung adenocarcinoma. Mod Pathol. 2018. https:// doi.org/10.1038/modpathol.2017.190.

10. Su G, Meyer K, Nandini CD, et al. Glypican-1 is frequently overexpressed in human gliomas and enhances FGF-2 signaling in glioma cells. Am J Pathol. 2006;168:2014-26.

11. Matsuzaki S, Serada S, Hiramatsu K, et al. Anti-glypican-1 antibody-drug conjugate exhibits potent preclinical antitumor activity against glypican-1 positive uterine cervical cancer. Int $\mathbf{J}$ Cancer. 2018;142:1056-66.

12. Matsuda K, Maruyama H, Guo F, et al. Glypican-1 is overexpressed in human breast cancer and modulates the mitogenic effects of multiple heparin-binding growth factors in breast cancer cells. Cancer Res. 2001;61:5562-9.

13. Kleeff J, Ishiwata T, Kumbasar A, et al. The cell-surface heparan sulfate proteoglycan glypican-1 regulates growth factor action in pancreatic carcinoma cells and is overexpressed in human pancreatic cancer. J Clin Invest. 1998;102:1662-73.

14. Lu H, Niu F, Liu F, et al. Elevated glypican-1 expression is associated with an unfavorable prognosis in pancreatic ductal adenocarcinoma. Cancer Med. 2017;6:1181-91.

15. Saito T, Sugiyama K, Hama S, et al. High expression of glypican1 predicts dissemination and poor prognosis in glioblastomas. World Neurosurg. 2017;105:282-8.

16. Hara H, Takahashi T, Serada S, et al. Overexpression of glypican1 implicates poor prognosis and their chemoresistance in oesophageal squamous cell carcinoma. Br J Cancer. 2016;115:66-75. 